\title{
Optimized Risk-Aware Nomination Strategy in Demand Response Markets
}

\author{
Ana Radovanovic \\ Google, Inc. \\ Mountain View, CA \\ AnaRadovanovic@google.com
}

\author{
William D. Heavlin \\ Google, Inc. \\ Mountain View, CA \\ BHeavlin@google.com
}

\author{
Sila Kiliccote \\ SLAC National Accelerator Laboratory \\ Menlo Park, CA \\ $+1.510 .384 .1635$ \\ SilaK@SLAC.Stanford.edu
}

\begin{abstract}
When wholesale prices are high and/or when the electricity system reliability is jeopardized, utilities have programs that encourage their customers to shed loads; these programs are called demand response (DR). Most large energy consumers, for example the buildings comprising Google's Mountain View, CA campus, have some ability to control, and therefore reduce, their demand, and in this way contribute to stabilizing the grid. In this paper, we present a novel risk-aware nomination strategy in DR programs where the capacity commitments are made one monthahead. The proposed methodology gives special treatment to the variability in load response to DR signals, variability that stems from the uncertainty in month-ahead weather forecasts and the inherent unpredictability in load profiles. The framework incorporates an efficient procedure for statistically characterizing the drop in demand associated with thermostatically controlled, heating, ventilation and air conditioning (HVAC) loads in commercial buildings, as well as applying the derived models to nominate revenue-optimal bids. The methodology is reasonably generic and adaptable to different kinds of DR markets and customer demand profiles. By way of example, we work out the capacity nomination for the Capacity Bidding Program (CBP) offered by Pacific Gas and Electric Company (PG\&E) that Google participated in with a fraction of its buildings.
\end{abstract}

\section{CCS Concepts}

- Hardware $\rightarrow$ Power and energy; Power estimation and optimization;

\section{Keywords}

Demand response nominations, revenue optimal nomination, riskaware bids, capacity bidding

\section{INTRODUCTION}

Electric utilities build and maintain a finite capacity for generating energy. With too much capacity, they incur capital expense; with too little, they risk not fulfilling customer demand. In periods of high demand, the reliability of the electricity system can be jeopardized and the price for obtaining additional capacity

Permission to make digital or hard copies of all or part of this work for personal or classroom use is granted without fee provided that copies are not made or distributed for profit or commercial advantage and that copies bear this notice and the full citation on the first page. Copyrights for components of this work owned by others than ACM must be honored. Abstracting with credit is permitted. To copy otherwise, or republish, to post on servers or to redistribute to lists, requires prior specific permission and/or a fee. Request permissions from Permissions@acm.org.

BuildSys '16, November 16-17, 2016, Palo Alto, CA, USA

(c) 2016 ACM. ISBN 978-1-4503-4264-3/16/11 ..\$15.00

DOI: http://dx.doi.org/10.1145/2993422.2993430 correspondingly rises. Therefore, utilities offer incentives (through demand response programs) for their customers to drop loads when requested. Most large customers, for example, Google's Mountain View, CA (Google MtV) campus, have some ability for reducing their demand, and in this way contribute to a more stable electricity grid with lower average cost.

For commercial buildings, Pacific Gas and Electric (PG\&E) offers a demand response program called the Capacity Bidding Program (CBP). PG\&E hosts fixed-price options in which various commercial buildings participate as bidders. CBP, active in PG\&E's territory from May 1 through October 31, requires that the bidders (participating customers) specify their bids five business days before the beginning of each month. Each bid presents a power demand reduction commitment in $\mathrm{kW}$ and its duration, made either the day before or the day of the DR event. At the utility's request, the bidders are obliged to deliver their bid throughout their specified duration. In return, after each month, CBP returns a payment that consists of a capacity payment $(\$ / \mathrm{kW})$ and energy payment $(\$ / \mathrm{kWh})$, where the latter are usually a small fraction of the former [1].

CBP is deliberately structured to shift risk from the utility to the bidding customers: (a) As noted above, customers' bids must be placed a month-ahead. (b) The utility decides when to define a DR event, with at most one day notice. (c) CBP's calculated demand reduction is relative to the utility-calculated baseline. To calculate this baseline, the utility averages each hour over the previous 10 days. Sometime the average of the past 10 days is much lower (or higher) than the actual consumption on the day of the DR event. So, customers are allowed to adjust their baseline based on the demand prior to the DR event.. Note that this baseline is calculated after the fact, and it does not incorporate local weather conditions, but does adapt to the current day's demand. These conventions shift a certain kind of forecast uncertainty onto the bidders. (d) The capacity incentive payments, described in Figure 1 below, are capped for over-performance, thus limit the utility's risk. Under-performance is severely penalized.

As a result of such risk shifting, it is appropriate for bidders to adapt their bids to the risk environment that CBP so deliberately constructs. This is the principal focus of this paper.

In the summer of 2015, Google participated in PG\&E's CBP with 20 buildings split into four groups. Google MtV buildings are quite different in their peak power demand, which ranges between approximately $30 \mathrm{~kW}$ and $600 \mathrm{~kW}$. The program spans the summer season between May 1st and October 31st, and almost all events fell on the warmest days of the season. In the particular case of Google $\mathrm{MtV}$ buildings, the response to an event notification consists of changing the DR level (from 0 to 1 or 2 ; level 1 response strategies being less severe than level 2), defined by temperature set point and its allowable range, throughout the 
duration of the event. When the DR event ends, the DR level is set to the default one, specified by the range of lower temperatures.

The key question for Google is identifying the revenue optimal bid (i.e., capacity nomination or demand reduction commitment) for each month (Section 2). The core component of this optimization is the model of load drop (i.e. response) during DR events for each DR group. In this paper, we propose a novel methodology for capturing the randomness in month-ahead nomination procedure, and its effect on building response models, in simulating monthly sample paths using Monte-Carlo framework (Section 3). The sample paths are mapped to the corresponding capacity payment outcomes using the defined, nonsmooth, capacity payment function defined by the CBP program. The generated payment samples allow us to propose risk-aware strategies for selecting the CBP bidding (nomination) strategy. Results of our empirical analysis are included in Section 4.

The choice of a building's DR response models needs to incorporate the fact that buildings have both diverse characteristics, and that integrated complex Building Management Systems (BMS) respond to control of temperature, ventilation and pressure in all zones simultaneously. All the approaches for modeling a building's response to changes in allowable temperature bands fall into two main categories: physical and statistical. Despite the substantial work on detailed physical models used to estimate building's DR potential [3], [4], [5], [6], [7], they commonly result in low prediction accuracy when exposed to a limited event history. In this paper, we propose an efficient statistical model for learning the response using a limited history of DR events, and a significantly larger history of nonevent, baseline, data (see Section 3). Even though there are learning techniques used for load forecasting, predicting user comfort levels and security of smart grids [23], [25], [26], to the best of our knowledge, there is almost no statistical treatment of the response inference problem when the number of DR events is small.

Monte-Carlo simulation framework is commonly used in cases where modeling and the impact of diverse and dependent random effects are hard to capture in an analytically tractable form. To this end, there is work that uses Monte-Carlo simulated sample paths for a large EV fleet, which is then fed into a scheduler to produce optimal coordination mechanisms of the designated aggregator (see, for example, [22]). Furthermore, this simulation approach can be used to learn the overall uncertainty in the demand across diverse distribution networks [20], or the impact of random investments in distributed generation [21]. In this paper, we propose using the Monte-Carlo simulation approach in order to quantify the uncertainty in the payments that are the result of bidding in DR markets.

To the best of our knowledge, this is the first methodology that unifies a careful, statistically efficient, treatment of uncertainty in a month-ahead demand response (CBP) program, and a strategic, risk-aware procedure for selected the revenue optimal bid nominations.

\section{RISK-AWARE NOMINATIONS IN DR MARKETS}

Each DR program has its payment that is a function of performance, i.e., the measured drop with respect to a corresponding baseline during event hours within a given month. In addition, other factors, such as month of the year, type of the program (day-ahead or day-of), etc., play a role in the definition of the payment. To this end, suppose that for a specific month, the total duration of all DR events was $H$ hours, and use $D_{1}, \ldots, D_{H}$ to denote drops corresponding to these DR event hours.

The payment is based on monthly performance and, for the majority of DR programs, consists of capacity and energy payments. After each month, the capacity payments are computed from measured drops (realizations of $D_{l}, \ldots, D_{H}$ ), and the payment structure reflects the specific time of the year and the details of the program.

We focus on the capacity payment for two reasons: (1) The magnitude of energy payments are small, at least relative to the capacity payments. (2) The complexity of forecasting energy payments is large. In part, they depend on real-time energy prices (locational marginal prices, LMPs); accounting for these requires month-ahead predictions of LMPs.

In the case of CBP, detailed in [18], the capacity payment is based on the delivery ratio. Just before each month, a certain DR capacity, say $\Gamma$, can be nominated. The performance achieved during DR events relative to the nominated capacity is the delivered capacity ratio, i.e., $D_{0} / \Gamma, D_{I} / \Gamma, \ldots, D_{H} / \Gamma$. The payment or penalty is based on this delivered capacity ratio as shown in Figure 1 below.

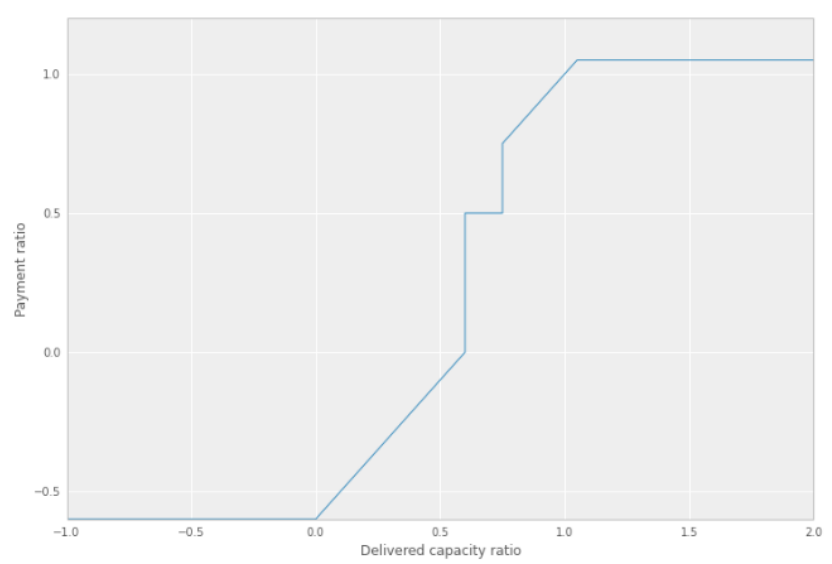

Figure 1. Capacity Bidding Program (CBP) - Payment ratio vs delivered capacity ratio.

Only in the narrow range between 0.75 and 1.05 is the payment ratio the same as the delivered ratio. Above that value, the payment clamps at 1.05 no matter how much drop is delivered. Below 0.75 , the payment drops to 0.5 for delivered ratio above 0.6. Below 0.6, there is an ever-increasing penalty, which caps at 0.6. As we can see, under-delivery is penalized much more than over-delivery is rewarded. Best performance is achieved if delivered capacity is right around nominated capacity. As noted above, this scheme of payment ratios functions to shift uncertainty from the utility to the CBP-participating customer.

Given the delivered capacity ratio for hour $h, l \leq h \leq H, D_{h} / \Gamma$, and the CBP payment ratio provided in Figure 1, the corresponding capacity payment, $Y\left(D_{h}, \Gamma\right)$, is computed as

$Y\left(D_{h}, \Gamma\right)=\frac{\Gamma \times(\text { Capacity price })}{\text { Number of Event Hours } H} \times$ Payment ratio $\left(D_{h} / \Gamma\right),(0)$

where (Capacity price) depends on the program type (day-of vs. day-ahead), as well as the month of the year (highest in August, lowest in October), and (Number of event hours H) is capped at 30 . For the specific month, the total capacity payment equals 


$$
\sum_{h=1}^{H} Y\left(D_{h}, \Gamma\right) .
$$

Customer's performance is observed only after the fact, and the capacity nomination needs to be submitted before the start of the specific month. Our analysis treats drops as random variables (Section 3), and uses the developed prediction models within the novel Monte-Carlo simulation framework to generate their monthahead realizations or sample paths. The proposed simulation approach allows us to analyze the statistical properties of the resulting capacity payments, as an explicit function of the nominated value. The result of one such simulation that is based on the analysis of a group of buildings on Google's MtV campus participating in the CBP program is captured in Figure 2.

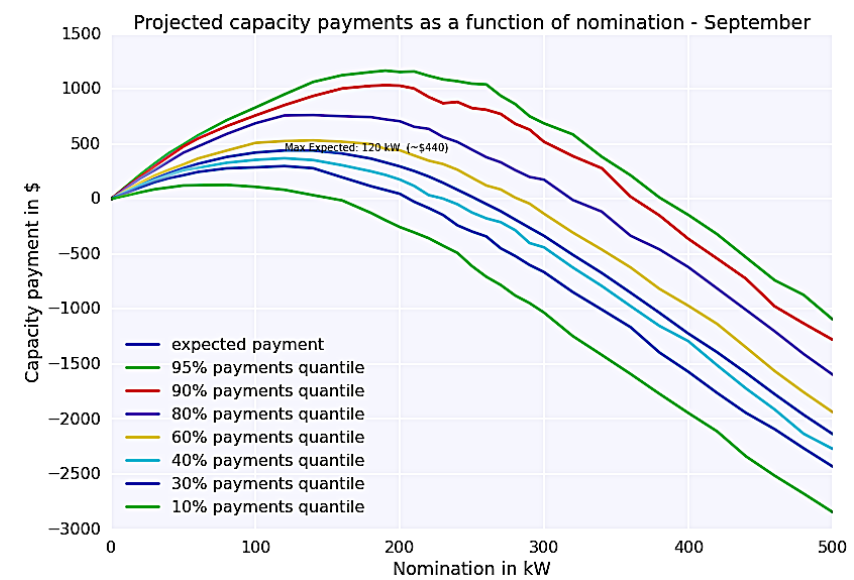

Figure 2. Quantile graph of capacity payments as a function of the nominated value.

Next, we propose a pair of potential optimization formulations that provide guidelines for selecting a risk-aware nomination subject to customer's ability to handle variation in the received payments. All the examples below are based on the analysis using the group of buildings on Google's MtV campus.

\subsection{Risk-averse Nomination Strategy}

Due to the uncertainty in the resulting payment, selecting the optimal nomination is not straightforward. As illustrated in Figure 3 , there is a value that maximizes the expected payment, but also implies a non-significant amount of variability that may or may not be tolerable. As the figure suggests, (1) intermediate bids lead to positive payments on average, (2) bidding larger values always leads to higher risk, and (3) therefore bidding at the highest values leads to diminished and ultimately negative payments.

Here, we propose two optimization formulations customers can use to come up with the optimal risk-averse nomination value. In the first one, we express customer's proneness to risk in terms of their susceptibility to the variation in the received payment. In the second case, customers can specify the desired likelihood of exceeding some specified payment.

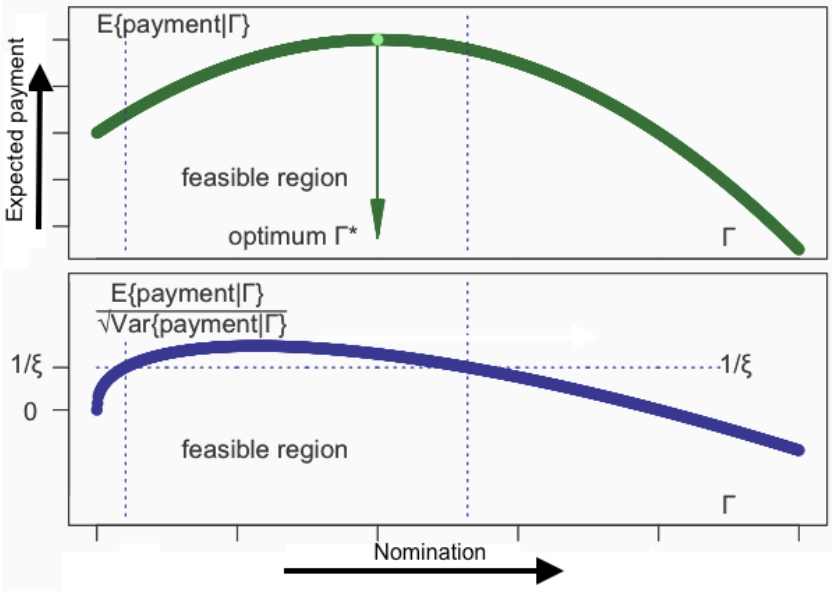

Figure 3. Conceptual explanation for the tradeoffs involved in risk-constrained optimal nomination: expected payment (top) and the associated risk (bottom) as a function of nomination.
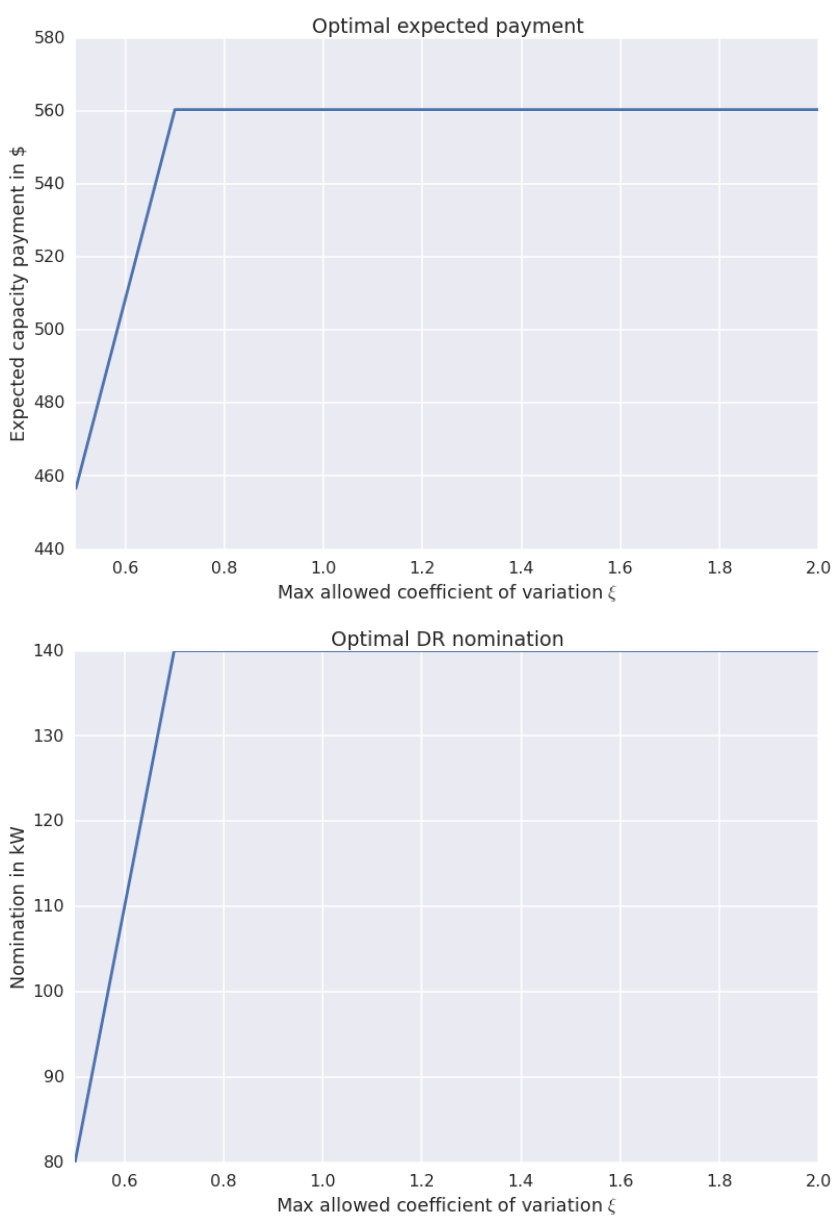

Figure 4. Optimal nomination and resulting payment as the function of customer's proneness to risk expressed through the maximum tolerable variation in the capacity payment. 
(I) The first proposed formulation finds the drop nomination $\Gamma$ that maximizes the expected capacity payment, while respecting customer's proneness to risk:

$\max _{\Gamma} \mathrm{E}[\mathrm{Y}(\Gamma)]$

s.t. $\sqrt{\operatorname{Var}[Y(\Gamma)]} \leq \xi \mathrm{E}[\mathrm{Y}(\Gamma)]$,

for some given $\xi$ which represents customer's proneness to risk, i.e., the smaller it is the more sensitive the customer is to the variability in payments. Figure 4 captures the result of solving (1) for different values of $\xi$. It is shown how the optimal expected payment and the corresponding DR nomination increase as the tolerance to risk gets larger until a certain point, when they become insensitive to it and keep the same value (560\$ corresponding to DR nomination of $140 \mathrm{~kW}$ ). As illustrated in Figure 4, this formalization constrains the feasible region of relatively moderate nominations.

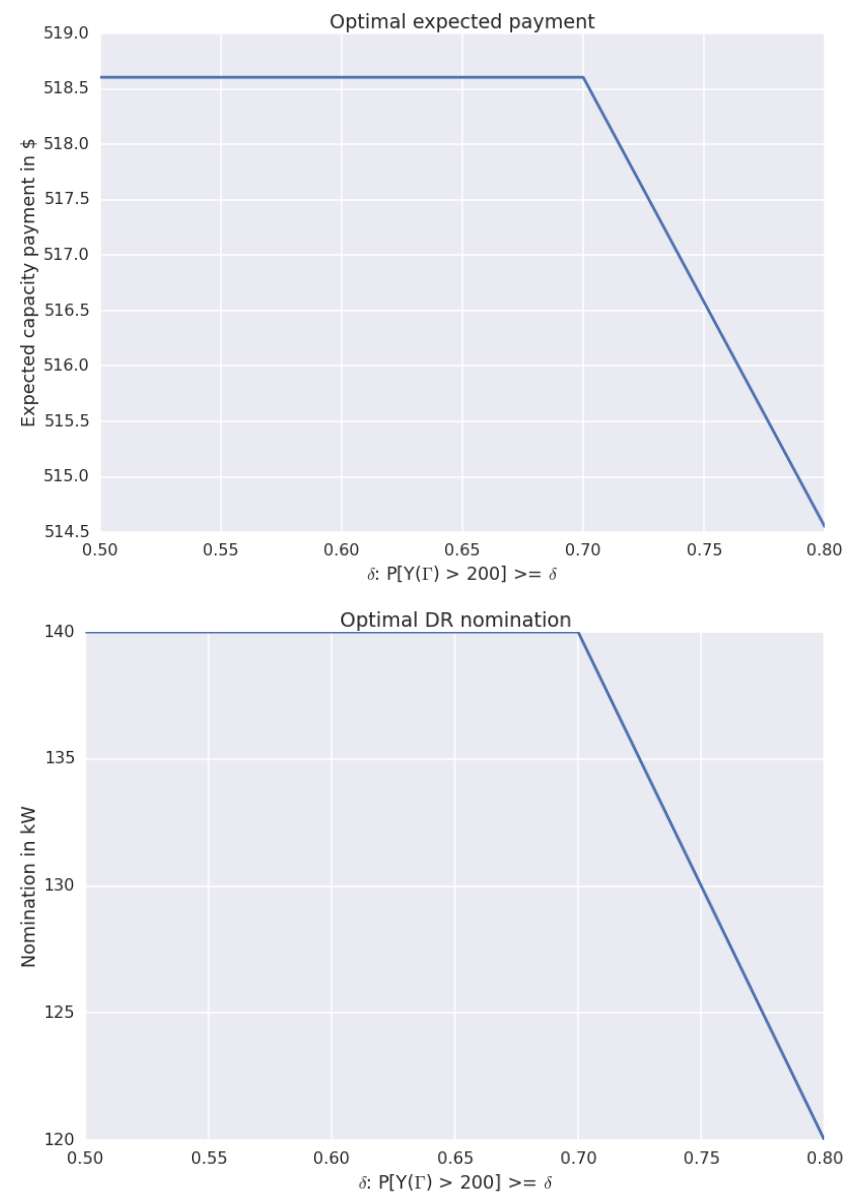

Figure 5. Optimal expected capacity payment and nomination value as the function of the payment level guarantees expressed by parameter $\delta \in[0,1]$.

(II) The second proposed formulation maximizes the expected capacity payment, while providing guarantees that the payment exceeds a given value $\bar{P}$ :

$\max _{\Gamma} \mathrm{E}[\mathrm{Y}(\Gamma)]$

s.t. $\mathbb{P}[\mathrm{Y}(\Gamma)>\bar{P}] \geq \delta$,

where $\delta$ and $\bar{P}$ are set, quantile-based, guarantees. Figure 5 shows that increasing $\delta$ (reducing the tolerance) constrains the feasible region in (2) and results in smaller optimal expected payments and corresponding nomination values.

\subsection{Performance of New Framework}

We used our methodology on two DR building groups, we call "Group 1" and "Group 2", on Google's MtV campus participating in the CBP program, and submitted the actual CBP nominations for months of August and September. The estimated drops for August and September were based on 10 (June and July) and 13 (June, July and August) DR events, respectively. For both months and groups, we submitted the nominations that maximize the expected capacity payment. The performance results are summarized in Table 1. For Group 1 that consists of buildings with more predictable power profiles, the expected capacity payment was 4\% higher (August) and 10\% (September) lower than the actually received one. On the other hand, Group 2 consists of less predictable buildings, and the expected payments were significantly lower than the actually received ones, $-50 \%$ (August) and $-200 \%$ (September). On closer examination, these large errors can be attributed to a single event (outlier) that skewed the trained model. Due to a very few events (data points) to learn from, a single outlier event could not be explained and is removed from the evaluation. When we remove this event, the expected payments are $-5 \%$ lower than the actually received ones. Increasing the collection of the past DR events should improve our predictions of demand drop, and, thereby, reduce the errors associated with any capacity nomination.

Table 1 The percent difference between the expected and the actually received payments for Groups 1 and 2 (negative difference means that we expect lower capacity payment).

\begin{tabular}{|l|l|l|l|}
\hline & Group 1 & Group 2 & $\begin{array}{l}\text { Group 2 } \\
\text { without } \\
\text { outlier(s) }\end{array}$ \\
\hline August & $+4 \%$ & $-50 \%$ & $-5 \%$ \\
\hline September & $-10 \%$ & $-200 \%$ & $-5 \%$ \\
\hline
\end{tabular}

In view of the quantile representation of the simulated capacity payments in Figure 2, and the discussion in Subsection 2.1, nomination decisions depend on the susceptibility to risk. Submitting nominations that maximize the expected capacity payment is less "risky" than nominating higher values that result in potentially higher payments, but with smaller probability. Before using the model-based approach to submit DR nominations, Google's tendency had been to bid the historical average of the reported DR drop for each group. These average drop values would generally be higher than the ones submitted in August and September using the model-based approach, which resulted in capacity payments that were sometimes significantly higher, and sometimes significantly lower that the expected ones. To that end, using the traditional approach to submit nominations for "Group 1" and "Group 2" in August and September, would have resulted in more than $10 \%$ smaller capacity payments.

\section{MONTE-CARLO SIMULATION FRAMEWORK}

In this section we outline the models that represent the components of the simulation framework used to generate samples of responses (drops) to DR events a month-ahead. We generate $\mathrm{N}(\mathrm{N}>200)$ monthly sample paths, indexed by $\mathrm{i}$, $\mathrm{i}=1,2, \ldots, N$, where path $i$ is described by $H$ hourly drops, a tuple $\left(\mathrm{D}_{i 1}, \ldots, \mathrm{D}_{i \mathrm{H}}\right)$. By applying different potential nominations $\Gamma=\left\{\Gamma_{1}, \Gamma_{2}, \ldots\right\}$, we can thereby compute capacity payments 
$\mathrm{Y}_{i}\left(\Gamma_{k}\right)=\sum_{h=1}^{H} Y\left(\mathrm{D}_{i h}, \Gamma_{k}\right), 1 \leq i \leq N$, for each nomination $\Gamma_{k} \in \Gamma$, which we then use to characterize their statistical properties. Figure 5 presents the result of one such analysis.

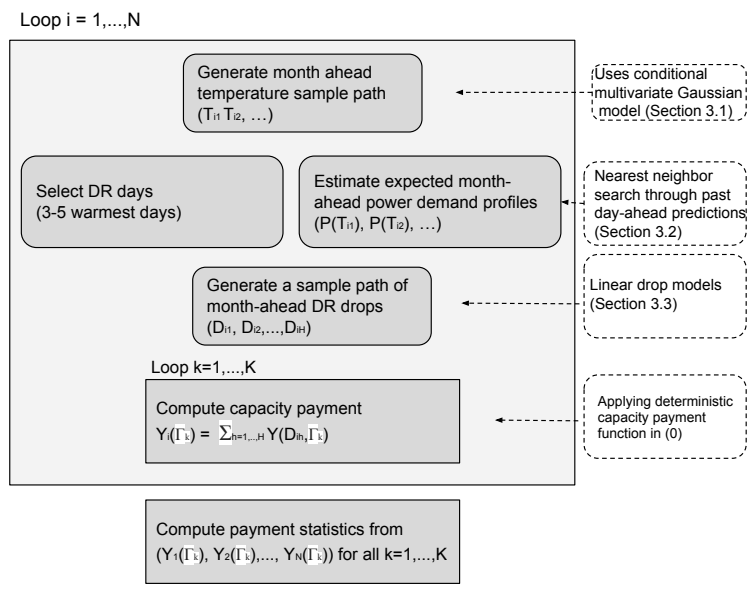

Figure 6. Monte-Carlo simulation architecture.

The components of the proposed simulation framework are captured by the architecture in Figure 6. Responses to DR events depend on the outside temperature, as well as the intrinsic uncertainty in buildings' response to changes in thermostat set points. To that end, Subsections 3.1-3.3, describe the proposed models that we train and apply in generating the sample paths of DR event responses for the observed building group.

\subsection{Generating Month-Ahead Temperature Profiles}

As captured in the flow diagram in Figure 6, a single simulation, indexed by $i$, consists of a vector of a month-ahead hourly temperatures (sample path), expressed by 24-dimensional, daily temperature profiles We assume that these daily profiles follow a 24-dimensional multivariate normal distribution, where we use their historical values between June and July of 2015 to estimate its mean and covariance matrix.

To generate a month-ahead sample path (e.g. for August 2015), we condition on daily peak and low temperatures, denoted by $\pi(d)$ and $\ell(d)$, respectively for day $d . \pi(d)$ and $\ell(d)$ are subject to the forecasting noise estimated from the monthly forecasts available at AccuWeather.com; the month-ahead forecasting noise is extrapolated using Google's internally available weather collection service (see Figure 7(b)). More specifically, starting from the first day, $d=1$, of the month, we make sure that the selected profiles drawn from the fitted distribution satisfy

$\left(\pi(d)-\theta_{d}, \pi(d)+\theta_{d}\right)$ and $\left(l(d)-\theta_{d}, l(d)+\theta_{d}\right)$,

where $\theta_{d} \sim N\left(0,(0.2 d)^{2}\right)$. The forecasting noise is drawn from the Gaussian distribution with mean 0 , and standard deviation 0.2 $\mathrm{d}$, where the coefficient of the error term, $0.2{ }^{\circ} \mathrm{C} / \mathrm{day}$, is extrapolated using Google's collection of actual temperatures and their forecasts. A sample of daily temperature profiles for a month ahead is shown in Figure 7(a).

Using historical CBP events, it can be learned that they are called on the warmest days in a month. In our case study, we had only 3 months of the CBP event data: 5 events in both June and July and 3 events in August. All of the events, except for one, lasted 4 hours each, on the warmest days of the month. Therefore, without losing too much generality, our simulation makes two assumptions about DR event intensity: (a) $H \in\{12,16,20\}$ hours, corresponding to 3, 4, and 5, 4-hour events, respectively, and (b) the events occur on the warmest days of the simulated monthly temperature profile. Assumptions (a) and (b) can be modified to consider more events, and/or different DR event drivers. In more general contexts of other DR programs, one can learn the event frequency, duration, correlations to daily temperature patterns from recent weather history, and use this to inform estimates of $H$.
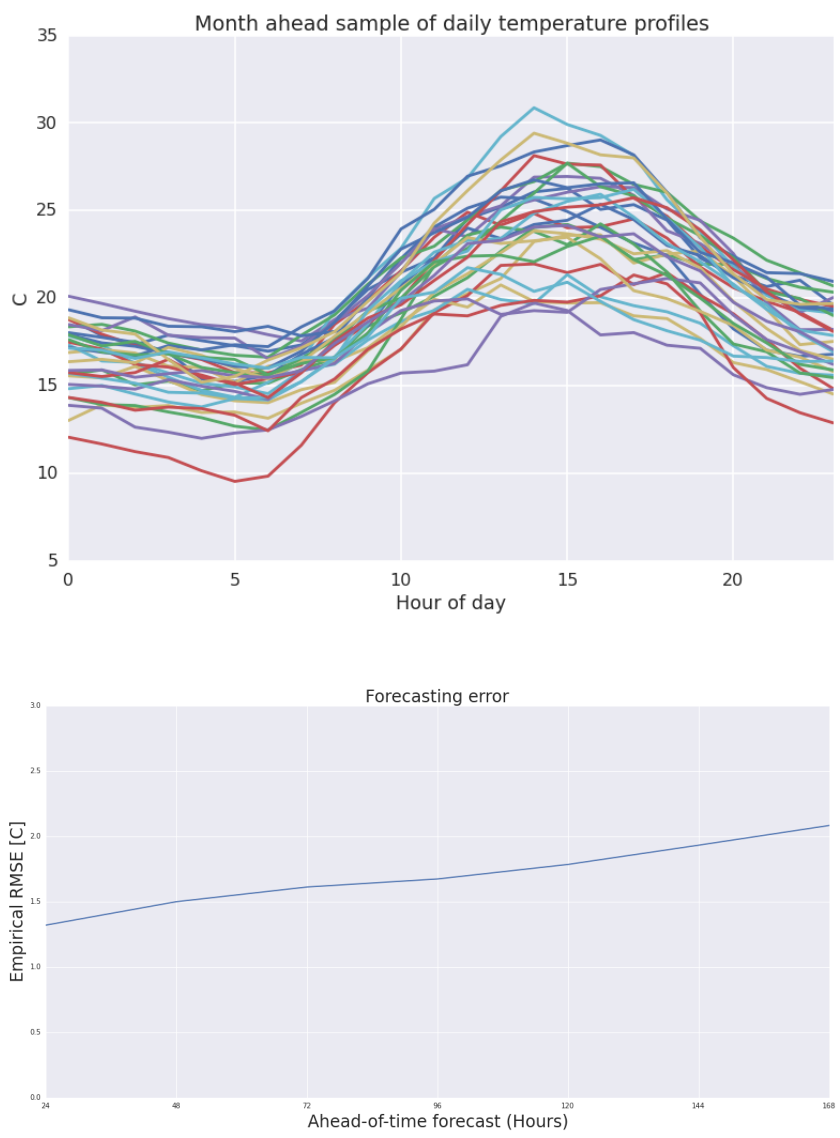

Figure 7. (a) An example of a month-ahead daily temperature profiles for August 2015. (b) Standard deviation of the temperature forecasting error as a function of time distance in the future.

Therefore, without losing too much generality, our simulation makes two assumptions about DR event intensity: (a) $H \in\{12,16$, 20 h hours, corresponding to 3, 4, and 5, 4-hour events, respectively, and (b) the events occur on the warmest days of the simulated monthly temperature profile. Assumptions (a) and (b) can be modified to consider more events, and/or different DR event drivers. In more general contexts of other DR programs, one can learn the event frequency, duration, correlations to daily temperature patterns from recent weather history, and use this to inform estimates of $H$.

\subsection{Month-Ahead Power Predictions}

Our analysis (see Section 4) shows that DR drops correlate with temperature and predicted power consumption during event hours. Hence, we develop a procedure for predicting month-ahead power consumption using generated month-ahead temperature sample paths (as described in Section 3.1). Our approach uses the 
nearest-neighbor-based search through the past day-ahead prediction profiles.

Past day-ahead prediction profiles are binned based on the corresponding daily temperature profiles and day of week. Suppose that $\left(\mathrm{T}_{i 1}, \mathrm{~T}_{i 2}, \ldots \mathrm{T}_{i \mathrm{~d}}, \ldots, \mathrm{T}_{i 31}\right), d=1, \ldots, 31$, represent a sample of daily temperature profiles for August. Next, suppose that there are bins that contain daily power prediction profiles for Mondays in the past (from May to July), and are denoted as $P^{\text {Mon }}\left(\widehat{T_{k}}\right), 1 \leq$ $k \leq K$, where $K$ is the selected number of temperature bins, and $\left(\widehat{T_{k}}\right)$ is a daily temperature profile representing the "key" of bin $k$. Analogously,

$\mathrm{U}_{k} P^{\text {Tue }}\left(\widehat{T_{k}}\right) \mathrm{U}_{k} P^{\text {Wed }}\left(\widehat{T_{k}}\right), \ldots, \mathrm{U}_{k} P^{\text {Fri }}\left(\widehat{T_{k}}\right)$ captures all of the past power predictions for other days of week, from May to the end of July. Then, for any specific day in August, say Wednesday, August 19th, we predict the power as

$\frac{\sum_{P \in P \text { Wed }\left(\widetilde{T_{k^{*}}}\right)} P}{\mid P^{\text {Wed }}\left(\widetilde{\left.T_{k^{*}}\right) \mid}\right.}$,

i.e., average power profile across all predictions within the selected bin $k^{*}$, corresponding to the "closest" match to $\mathrm{T}_{19}$, based on Euclidean norm during peak (DR likely) hours of day,

$$
k^{*}=\operatorname{argmin}_{k} \sum_{\text {peak hours } h}\left\|T_{19}(h)-\widehat{T_{k}}(h)\right\| .
$$

Our August 2016 nomination strategy uses only the 3 months available of past predictions, from May to July, resulting in at most 12 predicted power profiles for each business day of a week. By allocating a bin to each predicted power profile (i.e. no temperature-based clustering) and doing simple matching based on the generated temperature sample path, our after-the-fact analysis shows that month-ahead prediction errors turn out to be two times larger than the actual day-ahead prediction errors. We expect that, by having a longer history of day-ahead power prediction profiles, especially on warm days, the above-mentioned error would decrease significantly.

The framework in this paper does not depend on the particular approach for predicting day-ahead baselines. The approach reported here uses Multivariate Adaptive Regression Splines [8], and, in particular, Enhanced Adaptive Regression Through Hinges [9], due to their multiple benefits, such as simplicity, accuracy, flexibility, and interpretability.

\subsection{DR Drop Modeling}

Let $A(t), B(t), P(t)$ denote actual power, utility-defined baseline and predicted baseline at hourly time intervals $t \in\{1, \ldots, 24\}$, respectively. We select the time granularity to be one hour because the language of the DR program's nomination and monetization is in hours. $A(t)$ represents the sum of average hourly power consumptions across buildings in the selected DR group. Similarly, $B(t)$ and $P(t)$ are likewise the hour-indexed utility-defined and predicted baselines, and are computed by summing the corresponding quantities across buildings in the group. An example of a single day's actual power profile, with the corresponding predicted and utility-defined baselines is represented in Figure 8.

Let $D R(t) \in\{0,1\}$ denote a DR state (specified by the target temperature band) of a building at time $t: D R(t)=0$ is a default temperature band, while $D R(t)=1$ is used to denote a higher target temperature band, which leads to reducing a building's power consumption during a DR event.

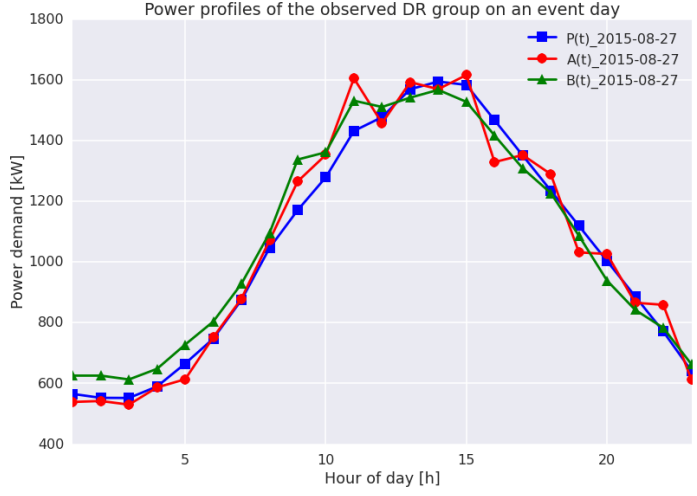

Figure 8. Aggregate daily power profiles for the selected DR group.

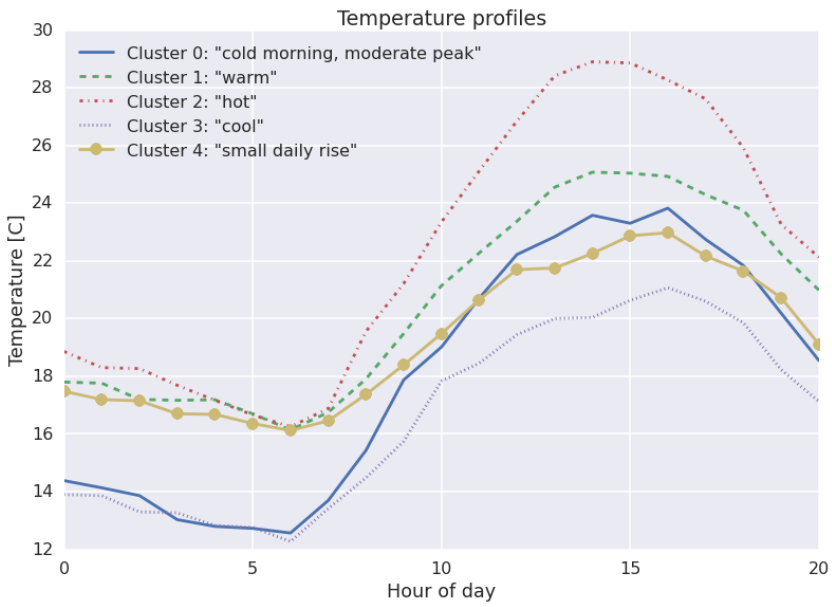

Figure 9. Typical daily temperature profiles identified using principle component analysis.

As described earlier, a customer's (in this case, Google's) performance in a DR program is based on its ability to drop load with respect to the utility-defined baseline during DR events throughout the one-month nomination period. In view of the above notation, the drop at time interval $t$ of a DR event day, can be expressed as

$\mathrm{D}(\mathrm{t}) \equiv B(t)-A(t)=(B(t)-P(t))+(\mathrm{P}(\mathrm{t})-\mathrm{A}(\mathrm{t})) \ldots$ for $\{t: \mathrm{DR}(t)=1\}$

Expression (3) has the same form as "average treatment effect of the treated" (ATT) [19]. One of the key challenges in the presented work lies in coming up with a representative model for a random drop, $\mathrm{D}(t)$, at the left hand side of (3). In particular, a special attention needs to be placed when capturing the inherent uncertainty in these models, which results from (i) the inability of the utility-defined baseline to accurately capture building's power consumption, and (ii) a typically small number of historical DR events from which we learn.

Table 2 illustrates the inherent uncertainty when estimating drop with respect to the utility-defined baseline. We compute the expected drop and its coefficient of variation $(100 \times$ standard deviation divided by expected value) for a group of DRparticipating buildings participating in a DR program with relatively high event frequency. 
Table 2. Intrinsic variability of a drop with respect to utilitydefined baseline.

\begin{tabular}{|l|l|l|}
\hline & Hour 1 & Hour 2 \\
\hline Expected drop [\%] & 10.5 & 8 \\
\hline Percent deviation [\%] & 25 & $>50$ \\
\hline
\end{tabular}

\subsubsection{Predicting Difference Between the Baselines}

In order to estimate the difference between the utility-defined and predicted baselines on DR days (the first summand on the righthand-side of (3)), we use all of the available historical data.. Note that the difference between the two baselines does not depend on actual events, but on non-DR related specificities of days on which demand response events are called. We build the model for predicting the difference $B(t)-P(t)$ using a set of "DR-like" days. This characterization allows us to enlarge the data set, and therefore reduce estimation error. We apply Principal Component Analysis [10] to cluster daily temperature profiles (see Figure 9) represented as 24-dimensional vectors, and show that DR event days tend to belong to a subset of principal components.

We model the relative difference between the baselines, i.e. $B(t) / P(t)-1$, for days within PCA clusters with the actual event days, while excluding the event days. Our model is the linear function of temperature, i.e.,

$\frac{B(t)}{P(t)}-1 \approx \alpha T(t)+\varepsilon(t)$,

where $\varepsilon(t)$ denotes the unobserved Gaussian noise with zero mean. More details on the specific procedure for fitting the linear model, and the corresponding results are available in Section 4.1.

\subsubsection{Modeling DR Drop with Respect to Predicted Baseline}

Capturing uncertainty in predicting the DR response, depends on different factors: DR event frequency, data availability (e.g., power data, temperature), time of events, and, in the case of Google campus buildings, an operating regime of cooling loads, which depend significantly on the outside temperature and the various buildings' cooling capacities.

We find that the predicted baseline correlates with the percentage drop (see Section 4.2), motivating the following linear model:

$1-\frac{A(t)}{P(t)} \approx \gamma T+\beta P(t)+\eta(t)$,

where $\eta(t)$ denotes the Gaussian noise (i.e. prediction error), with zero mean.

\subsubsection{Relative DR Drop Estimation Error}

Previously, we described our prediction models for the two terms on the right hand side of (3). Here, we discuss our rationale for treating the two errors, $\varepsilon(t)$, and $\eta(t)$, as uncorrelated one from the other in the evaluation of the drop with respect to the utilitydefined baseline.

Using (4) and (5), the variance between the models of terms $B(t) / P(t)-1$ and $1-A(t) / P(t)$, can be expressed as

$$
E[\hat{\alpha} T+\hat{\varepsilon})(\hat{\gamma} T+\hat{\beta} P+\hat{\eta})]-E[\hat{\alpha} T+\hat{\varepsilon}] E[\hat{\gamma} T+\hat{\beta} P+\hat{\eta}]
$$

where $\hat{\alpha}, \hat{\beta}, \hat{\gamma}$, are estimated model coefficients, and $\hat{\varepsilon}$ and $\hat{\gamma}$ are the error terms for the two models. Note that our procedure estimates $\hat{\alpha}$ based on DR-like days, defining a set $\Phi_{D R-l i k e}$ of data points, while $\hat{\gamma}$ and $\widehat{\beta}$ are the estimates based on DR event data. Since $\Phi_{D R \text {-like }} \cap \Phi_{D R}=\oslash$, estimation noise $\hat{\varepsilon}(t)$ and $\hat{\eta}(t)$ are only weakly correlated, implying
$\operatorname{Var}(\mathrm{D}(t)) \approx \operatorname{Var}(\varepsilon(t))+\operatorname{Var}(\eta(t))$.

\section{RESULTS OF DATA ANALYSIS}

Ninety buildings on Google Mountain View campus are grouped into sub-Load Aggregation Points, sub-LAPs, (DR groups) and registered in several DR programs, including Capacity Bidding Program (CBP) and Supply Side Pilot (SSP). All of the buildings control the DR level of their HVAC systems to change their power consumption during DR events. The buildings vary in size and physical properties, implying significant differences in their power consumption and its predictability. Table 2 captures basic characteristics of a subset of buildings participating in the CBP program, and shows diversity in their peak power consumption and predictability at peak demand hours. Buildings 1 to 7 belong to a single sub-lap, say DR, and will be used to describe our methodology. Buildings 8 to 11 belong to a different sub-lap, and are used here to demonstrate the impact of aggregation into larger sub-laps: $D R^{*}=D R \cup\{8,9\}$, and $D R^{* *}=D R \cup\{10,11\}$.

Table 3. Basic properties of Google MtV buildings that are subject of our analysis.

\begin{tabular}{|l|c|c|}
\hline & $\begin{array}{l}\text { Average peak } \\
\text { during summer } \\
\text { months (kW) }\end{array}$ & $\begin{array}{l}\text { \% RMSE } \\
\text { prediction error } \\
\text { during peak } \\
\text { hours }\end{array}$ \\
\hline Building 1 & 214 & 13 \\
\hline Building 2 & 68 & 15.5 \\
\hline Building 3 & 77 & 8.5 \\
\hline Building 4 & 37 & 9.6 \\
\hline Building 5 & 456 & 11 \\
\hline Building 6 & 194 & 16.7 \\
\hline Building 7 & 216 & 11.4 \\
\hline Building 8* & 155 & 11 \\
\hline Building 9* & 596 & 14 \\
\hline Building 10** & 435 & 12 \\
\hline Building 11** & 170 & 6 \\
\hline
\end{tabular}

Actual power data represents average power consumption at $15 \mathrm{~min}$ time granularity, and is obtained from PG\&E. The average peak power is obtained by averaging $15 \mathrm{~min}$ power values between $3 \mathrm{pm}$ and $7 \mathrm{pm}$, which are CBP event times. In the case some missing data is detected, there is a new attempt to get the data of the specific day. Overall, the amount of missing data is negligible. Also, anomalies in the power series are rare, and are smoothed out using linearization techniques. To train our models for predicting power demand for each building, we use time series power data since January 1st, 2015. More specifically, our nomination strategy for August 2015 is based on power data between January $1 \mathrm{st}$, and, say, the day of nomination (July 26th, 2015). Analogously, nomination strategy for September 2015 is based on power data between January 1st till the end of August, etc.

\subsection{Calculating the Difference Between the Baselines}

In order to estimate the difference on DR days between the utilitydefined and predicted baselines on DR days, we use all the available historical data and event information on past DR events. Note that the difference between the two baselines does not depend on actual events, but on non-DR related predictors, and 
the specifics of days on which demand response events are called. In particular, our analysis shows that CBP events strongly depend on the outside temperature, and were called on a subset of the warmest days during the summer of 2015. Furthermore, all events except for one (on July 17th, 2015) lasted for four hours, between 3 and $7 \mathrm{pm}$.

In view of the above, we build the model for predicting the difference using a set of "DR-like" days. The binning procedure will allow us to enlarge the data set, and, therefore, reduce the estimation error. We apply Principal Component Analysis [10] to cluster daily temperature profiles. Our analysis showed that DR event days tend to belong to a subset of principal components (see Table 4).

Table 4. The result of Principal Component Analysis using daily temperature profiles throughout summer 2015. Dates in red are the actual DR event days. The set of DR-like days consists of the dates in bold.

\begin{tabular}{|c|c|c|}
\hline $\begin{array}{l}\text { PCA } \\
\text { cluster }\end{array}$ & $\begin{array}{l}\text { Cluster } \\
\text { name }\end{array}$ & List of dates \\
\hline 2 & "hot" & $\begin{array}{l}06 / 08,06 / 25,06 / 30,07 / 20,07 / 27, \\
07 / 28,07 / 29,08 / 17,08 / 27,08 / 28\end{array}$ \\
\hline 4 & "small & $\begin{array}{lllll}06 / 10, & 06 / 11, & 06 / 29, & 07 / 09, & 07 / 10, \\
07 / 14, & 07 / 21, & 07 / 22, & 07 / 23, & 07 / 30, \\
08 / 04, & 08 / 06, & 08 / 19, & 08 / 21, & 08 / 24, \\
08 / 25 & & & & \end{array}$ \\
\hline 3 & "cool" & $\begin{array}{lllll}04 / 29, & 06 / 09, & 06 / 12, & 06 / 26, & 07 / 01, \\
07 / 02, & 07 / 03, & 07 / 06, & 07 / 13, & 07 / 15, \\
07 / 16, & & & \\
07 / 17, & 07 / 30, & 08 / 03, & 08 / 05, & 08 / 07, \\
08 / 10, & 08 / 11, & 08 / 12, & 08 / 13, & 08 / 14, \\
08 / 18, & & & \\
08 / 26,08 / 31 & & & \end{array}$ \\
\hline 0 & $\begin{array}{l}\text { "cold } \\
\text { morning, } \\
\text { moderate } \\
\text { peak" }\end{array}$ & $\begin{array}{l}04 / 15, \quad 04 / 28,06 / 02,06 / 03,06 / 15 \\
06 / 16,06 / 22\end{array}$ \\
\hline 1 & "warm" & $\begin{array}{l}04 / 16, \quad 04 / 17,04 / 27,06 / 04, \quad 06 / 05 \\
06 / 18,06 / 19,06 / 23,06 / 24,07 / 24\end{array}$ \\
\hline
\end{tabular}

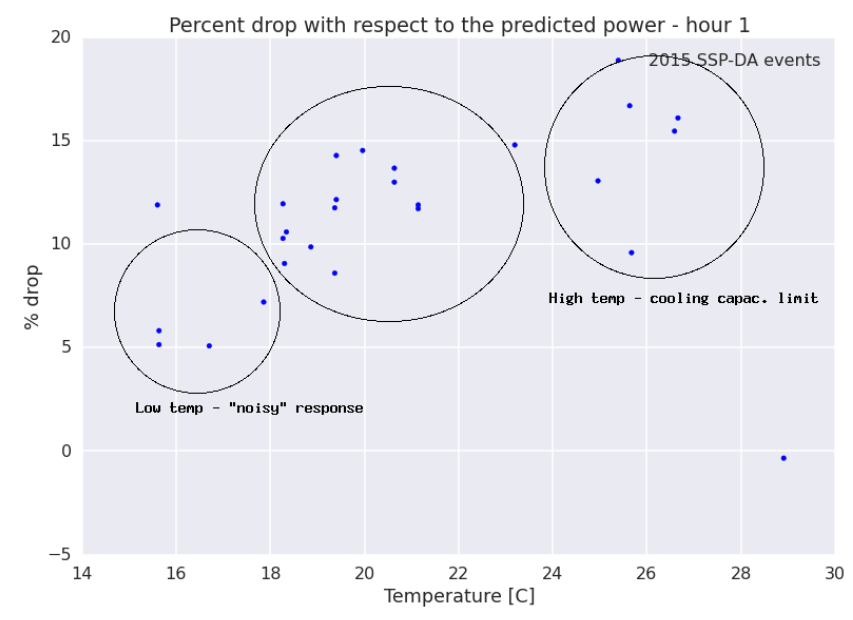

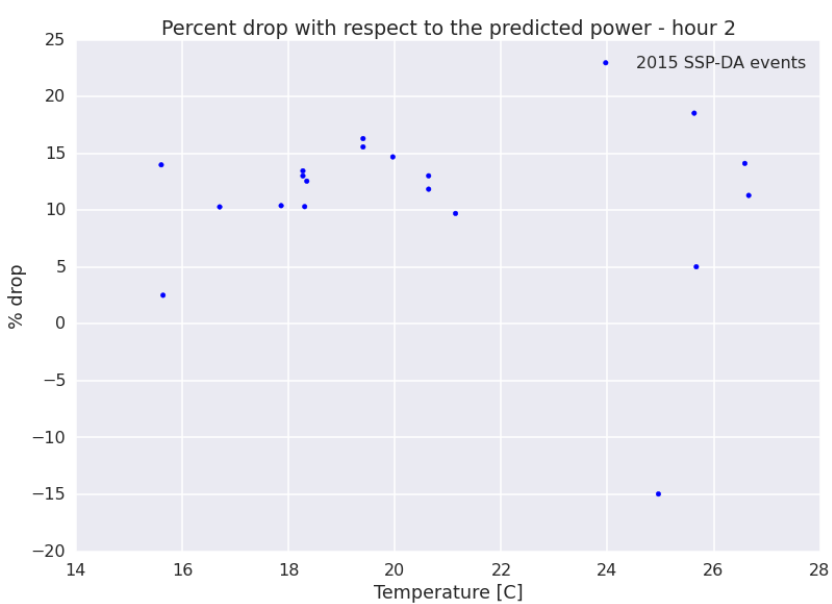

Figure 10. Different drop ability as the function of the outside temperature.

We fit the model in (4) using the re-weighted linear regression model to reduce the impact of outliers. Table 5 below contains the estimation error for predicting the relative difference between the two baselines.

Table 5. Root Mean Squared Error [\%] in predicting the relative difference between the utility-defined and predicted baselines for each hour of an event.

\begin{tabular}{|l|l|l|l|l|}
\hline Estimation Error & Hour 1 & Hour 2 & Hour 3 & Hour 4 \\
\hline RMSE $(\varepsilon(\mathrm{t}))[\%]$ & 8.2 & 8.2 & 9.6 & 10 \\
\hline
\end{tabular}

Table 6 captures the impact of load size and predictability (by changing the set of buildings in a DR group) on the estimation accuracy of the model in (4). The examples show how the predictions in building power profiles can impact the estimation accuracy of their aggregates in different ways. Adding "noisy" buildings, 10 and 11 (in DR**), to the original DR group results in less predictable baselines, and, therefore, lower prediction accuracy. On the other hand, adding "less noisy" buildings, 8 and 9 (in $\mathrm{DR}^{*}$ ), improves the predictability of the whole group's baselines, and leads to the smaller prediction error.

Table 6. The impact of building groupings on the error in predicting the relative difference between the baselines.

\begin{tabular}{|l|l|l|l|l|}
\hline $\begin{array}{l}\text { \% Estimation } \\
\text { Noise }\end{array}$ & $3-4 \mathrm{pm}$ & $4-5 \mathrm{pm}$ & $5-6 \mathrm{pm}$ & $6-7 \mathrm{pm}$ \\
\hline DR* & 6.5 & 6.5 & 7.7 & 8.5 \\
\hline DR** & 9.2 & 9 & 9.7 & 10 \\
\hline $\mathrm{DR}$ & 8 & 8.2 & 9.6 & 10 \\
\hline $\mathrm{DR}-\{1,7\}$ & 10.8 & 11.2 & 12 & 13 \\
\hline
\end{tabular}

\subsection{Predicting Drop on DR Days}

Our approach in predicting the DR drop utilizes the information collected on the past DR days. DR programs differ in their frequency, and do not necessarily happen on the warmest days. The analysis of DR responses for DR groups participating in different programs identifies different cooling regimes that are a function of the outside temperature.

More specifically, in the analysis of responses from a similar, more frequent non-weather driven program, we observe a wide spectrum of cooling regimes in Google buildings. For some range of moderate temperatures, the load seems insensitive to 
temperature because cooling is not needed. Then, there is a regime of temperatures for which we detect a positive correlation between the observed DR drop and the outside temperature. Finally, for very high outside temperatures, the HVAC load reaches its maximum cooling capacity, and the DR response becomes less predictable (see Figure 10).
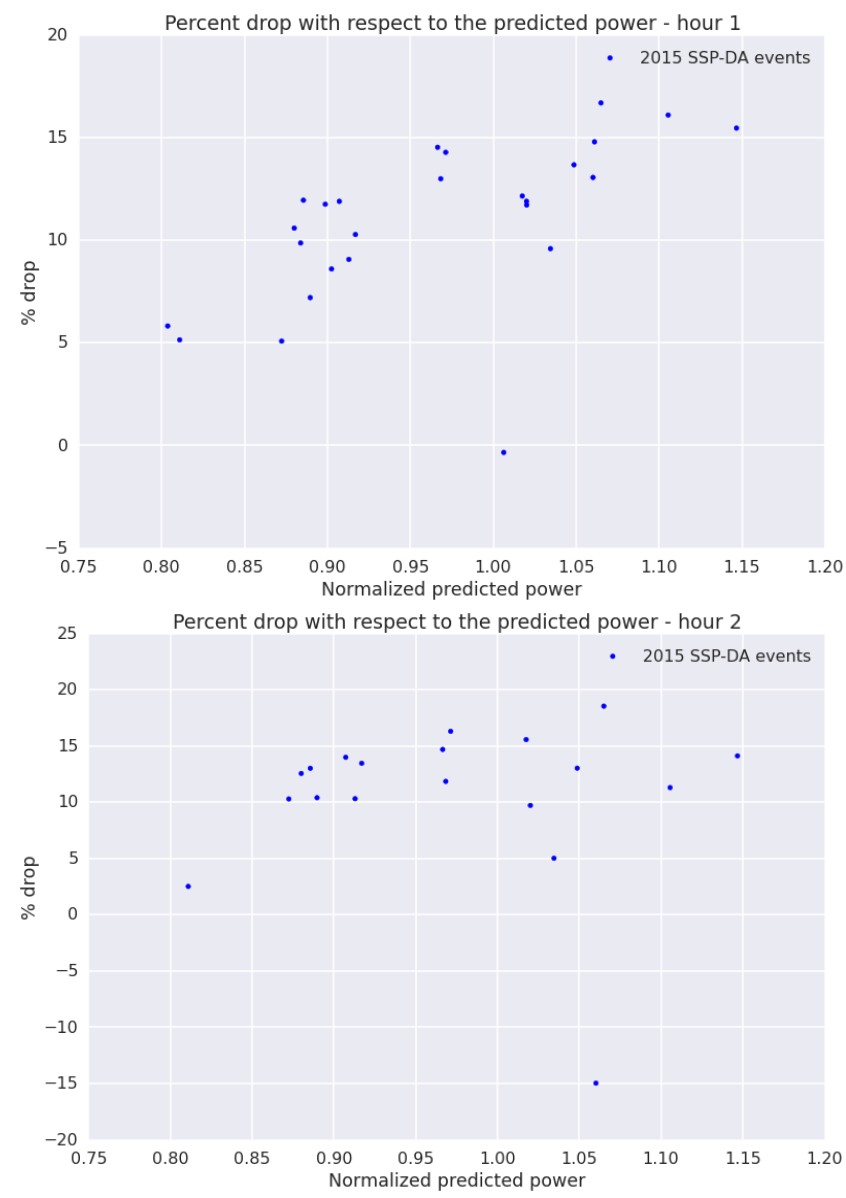

Figure 11. DR group participating in more frequent, nonweather-driven, DR events: percent drop as the function of the predicted power.

We find that, apart from depending on temperature, the percent drop with respect to the predicted baseline correlates with the predicted baseline as well (see Figure 11). Furthermore, CBP events are typically called on the warmest days, requiring the maximum cooling capacity of the analyzed buildings. Therefore, in view of the discussion above, the impact the temperature becomes

small.

Using the linear model in (5), we find that the linear weighted regression [11] model is an efficient way to capture the drop, remove the effect of outliers, and handle the small number of past DR events. Table 7 captures the obtained estimation accuracy.

Table 7. Root Mean Squared Error [\%] in predicting the drop with respect to the predicted baseline for each event hour.

\begin{tabular}{|l|l|l|l|l|}
\hline Estimation Error & Hour 1 & Hour 2 & Hour 3 & Hour 4 \\
\hline RMSE $(\varepsilon(\mathrm{t}))[\%]$ & 5.7 & 6 & 8 & 8.7 \\
\hline
\end{tabular}

Table 8 captures the impact of load size and predictability (by changing the set of buildings in a DR group) on the estimation accuracy of the DR drop. By aggregating loads, DR drop becomes a result of the superposition of loads' response to a DR event, leading to a smaller uncertainty in drop estimation.

Table 8. Adding buildings to a DR group reduces the drop prediction error.

\begin{tabular}{|l|l|l|l|l|}
\hline $\begin{array}{l}\text { \% Estimation } \\
\text { Noise }\end{array}$ & $3-4 \mathrm{pm}$ & $4-5 \mathrm{pm}$ & $5-6 \mathrm{pm}$ & $6-7 \mathrm{pm}$ \\
\hline DR* & 5.1 & 5 & 6.7 & 6.2 \\
\hline DR** & 4.9 & 5 & 7.2 & 8.6 \\
\hline DR & 5.7 & 6 & 8 & 8.7 \\
\hline $\mathrm{DR}-\{1,7\}$ & 6.1 & 7.5 & 8.7 & 9.4 \\
\hline
\end{tabular}

\section{CONCLUSIONS}

In this paper, we propose a novel risk-aware nomination strategy in DR programs, where the capacity commitments are made one month-ahead. The new procedure incorporates intrinsic randomness in the analysis of DR performance, and we validate it using the actual data from DR event participation in CBP. There are two optimization formulations used to obtain optimal riskaverse nominations. In the first one, we express customer's proneness to risk in terms of their susceptibility to the variation in the received payment. In the second case, customers can specify the desired likelihood of exceeding some specified payment. Our validation of the methodology compares the results of the analysis against the actual payments received as a result of Google's participation in the CBP. In the case of the more predictable building group, the expected capacity payment was $4 \%$ higher than the actual payment in August, and 10\% lower in September. In the case of the less predictable DR group, the expected payments were significantly lower than the actually received ones; $50 \%$ in August, and 200\% in September, due to a single event that turned out to be an outlier to the developed model. When we remove the outlier (i.e. unusual DR event outcome), the expected payments were $5 \%$ lower than the actually received ones. Our results show that increasing the data of the past DR events improves our drop predictions, and also reduces the error in the optimization procedure. Finally, we describe the prediction models that are building blocks of the proposed methodology, their performance in predicting the baseline, as well as summarize the response to DR event signals.

\section{REFERENCES}

[1] PG\&E, "Capacity Bidding Program", http://www.pge.com/tariffs/tm2/pdf/ELEC_SCHEDS_ECBP.pdf

[2] Coughlin, K., Piette, M.A., Goldman, C.A., Kiliccote S. Statistical analysis of baseline load models for nonresidential buildings. Energy and Buildings, Volume 41, Issue 4, April 2009. LBNL-4984E.

[3] Yin, R., Xu P., Piette M.A., Kiliccote S. Study on Auto-DR and Pre-cooling of Commercial Buildings with Thermal Mass in California. Energy and Buildings, 42, no. 7 (2010): 967-975.

[4] Kundu, S., Sinitsyn, N., Backhaus, S., Hiskens. I. Modeling and control of thermostatically controlled loads. 17th Power Systems Computation Conference, 2011.

[5] Bashash S. and Fathy. H.K. Modeling and control of aggregate air conditioning loads for robust renewable power management. IEEE Transactions on Control Systems Technology, 2013. 
[6] Shao, S., Pipattanasomporn, M. and Rahman, S Development of physical-based demand response-enabled residential load models. IEEE Transactions on Power Systems, vol. 28, no. 2, pp. 607-614, 2013.

[7] Yin, R., Kiliccote, S., Piette. M.A. Linking measurements and models in commercial buildings: A case study for model calibration and demand response strategy evaluation. 2014. LBNL-7006E.

[8] Friedman, J. H. Multivariate Adaptive Regression Splines. Annals of Statistics 19 (1991), no. 1, 1--67. doi:10.1214/aos/1176347963. http://projecteuclid.org/euclid.aos/1176347963.

[9] Milborrow, S. Derived from mda:mars by T. Hastie and R. Tibshirani. earth: Multivariate Adaptive Regression Splines. 2011. R package.

[10] Yeung, Ka Yee, and Walter L. Ruzzo(2001). Principal component analysis for clustering gene expression data. Bioinformatics, 17, 9, 763-774..

[11] Nelder, J. and Wedderburn, R.(1972). Generalized Linear Models. Journal of the Royal Statistical Society. Series A (General) 135 (3): 370-384. doi:10.2307/2344614

[12] Mathieu, J.L., Price, P.N., Kiliccote, S., and Piette, MA. Quantifying changes in building electricity use, with application in demand response. IEEE Transactions on Smart Grid, 2, 3, 507-518. 2011.

[13] James W. Taylor, Lilian M. de Menezes, Patrick E. McSharry (2006). A comparison of univariate methods for forecasting electricity demand up to a day-ahead. International Journal on Forecasting, 22, 1-16.

[14] Piras, A. and Buchenel. B. A tutorial on short term load forecasting. Engineering Intelligent Systems, 1, 41-47, 1999.

[15] D.E. Claridge (1998). A Perspective on Methods for Analysis of Measured Energy Data from Commercial Buildings. Journal of Solar Energy Engineering, 120, 3, 150155.

[16] Haberl, J.S., and Thamilseran, S.. Predicting Hourly Building Energy Use: The Great Energy Predictor Shootout II: Measuring Retrofit Savings: Preview and Discussion of Results, ASHRAE Transactions, 102, 2, 419-435.1996.

[17] Dhar, A. , Reddy, T.A. and Claridge, D. Modeling Hourly Energy Use In Commercial Buildings With Fourier Series Functional Forms. Journal of Solar Energy Engineering, 120, 3, 217-223. 1998.

[18] PG\&E, "Capacity Bidding Program", http://www.pge.com/tariffs/tm2/pdf/ELEC_SCHEDS_ECBP.pdf

[19] Imbens, G.W., and Joshua D. A.. Identification and Estimation of Local Average Treatment Effects. Econometrica 62.2 (1994): 467-475.

[20] McQueen, D. H. O., Hyland, P. R.,Watson, S. J. Monte Carlo Simulation of Residential Electricity Demand for Forecasting Maximum Demand on Distribution Networks. IEEE Transactions on Power Systems, Vol. 19, No. 3, August 2004.

[21] Mendez, V.H., Rivier, J., de la Fuente, J.I., Gomez, T., Arcelu, J., Marín, J., Madurg. A. Impact of distributed generation on distribution investment deferral. Electrical Power and Energy Systems 28 (2006) 244-252

[22] Sandels,C., Franke, U., Ingvar, N., Nordstrom, L., Hamren. R. Vehicle to Grid - Monte Carlo simulations for optimal Aggregator Strategies. 2010 International Conference on Power System Technology, 2010.

[23] Lei, Y., Zhang, J., Qian, D. Risk-aware Day-ahead Scheduling and Real-time Dispatch for Plug-in Electric Vehicles. IEEE Globecom 2012 - Symposium on Selected Areas in Communications.

[24] Zhang, H.-T, Xu, F.-Y., Zhou, L. Artificial neural network for load forecasting in smart grid. International Conference on Machine Learning and Cybernetics (ICMLC), 2010.

[25] Li, B., Gangadhar, S., Cheng, S., Verma, P. K. Predicting user comfort level using machine learning for smart grid environments. IEEE PES Innovative Smart Grid Technologies Conference, 2011.

[26] Ma, Y., Zhou, Tse, N., Osman, A., Lai, L. An initial study on computational intelligence for smart grid. International Conference on Machine Learning and Cybernetics (ICMLC), 2009. Bowman, M., Debray, S. K., and Peterson, L. L., 1993. Reasoning about naming systems. ACM Trans. Program. Lang. Syst. 15, 5 (Nov. 1993), 795-825. DOI= http://doi.acm.org/10.1145/161468.16147.

[27] Ding, W. and Marchionini, G. A Study on Video Browsing Strategies. Technical Report. University of Maryland at College Park, 1997.

[28] Fröhlich, B. and Plate, J. 2000. The cubic mouse: a new device for three-dimensional input. Proceedings of the SIGCHI Conference on Human Factors in Computing Systems (The Hague, The Netherlands, April 01 - 06, 2000). CHI '00. ACM, New York, NY, 526-531. DOI= http://doi.acm.org/10.1145/332040.332491.

[29] Tavel, P. Modeling and Simulation Design. AK Peters Ltd., Natick, MA. 2007

[30] Sannella, M. J. 1994. .Constraint Satisfaction and Debugging for Interactive User Interfaces. Doctoral Thesis. UMI Order Number: UMI Order No. GAX95-09398, University of Washington.

[31] Forman, G. 2003. An extensive empirical study of feature selection metrics for text classification. Journal of Machine Learning Research 3 (Mar. 2003), 1289-1305.

[32] Brown, L. D., Hua, H., and Gao, C. 2003. A widget framework for augmented interaction in SCAPE. In Proceedings of the 16th Annual ACM Symposium on User Interface Software and Technology (Vancouver, Canada, November 02 - 05, 2003). UIST '03. ACM, New York, NY, 1-10. DOI= http://doi.acm.org/10.1145/964696.964697.

[33] Yu, Y. T. and Lau, M. F. 2006. A comparison of MC/DC, MUMCUT and several other coverage criteria for logical decisions. J. Syst. Softw. 79, 5 (May. 2006), 577-590. DOI= http://dx.doi.org/10.1016/j.jss.2005.05.030.

[34] Spector, A. Z. 1989. Achieving application requirements. Distributed Systems, S. Mullender, Editor. ACM Press Frontier Series. ACM, New York, NY, 19-33. DOI= http://doi.acm.org/10.1145/90417.90738. 